\title{
Strapdown Inertial Navigation Algorithm Based on MIMU and Its Application in Missile Ejection Test
}

\author{
BIAN Wei-wei ${ }^{a}{ }^{*}$, XIN Zhen-fang ${ }^{b}$, ZHAO Jin-xin $^{c}$, LI Jia-hui ${ }^{d}$ \\ Beijing Machine and Equipment Institute, Beijing 100854, China

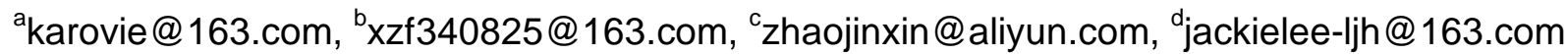

Keywords: MIMU; SINS; algorithm model; missile motion parameters

Abstract: A strapdown inertial navigation algorithm is proposed, which is suitable for the measurement of the parameters of missile in the ejection test with short movement time, small changes of velocity and position. The transformation relationship between the missile-boy coordinate frame and the navigation coordinate frame is described by the attitude matrix. The initial attitude angles of the missile are determined by the initial alignment processing static data. The navigation solution of missile motion parameters is realized by using the attitude update model, the velocity update model and the position update model. Numerical simulation results show that the proposed algorithm can calculate the position, velocity and attitude angles of the missile.

\section{Introduction}

The measure precision and stability of MIMU (micro inertial measurement unit) have become increasingly along with the continuous development of MEMS (micro electro mechanical system) technology. At present, with characteristics of smaller dimensions, lighter weight, longer use time, high reliability, lower cost and adaptive surrounding etc., MIMU has been used widely in the field of aviation and aerospace, automobile automation and biomedical engineering and so on [1,2]. A strapdown inertial navigation algorithm for missile injection test is put forward in this report.

\section{Navigation algorithm principle}

The algorithm of SINS calculates the position, velocity and attitude information of missile by using the data of the MIMU output after error compensation and coordinate transformation [3]. In the missile ejection test, neglecting the influence of the launching angle and the rotation of the earth will not cause obvious errors to the results because of short movement time, small changes of velocity and position. The strapdown inertial navigation algorithm is shown in Fig.1.

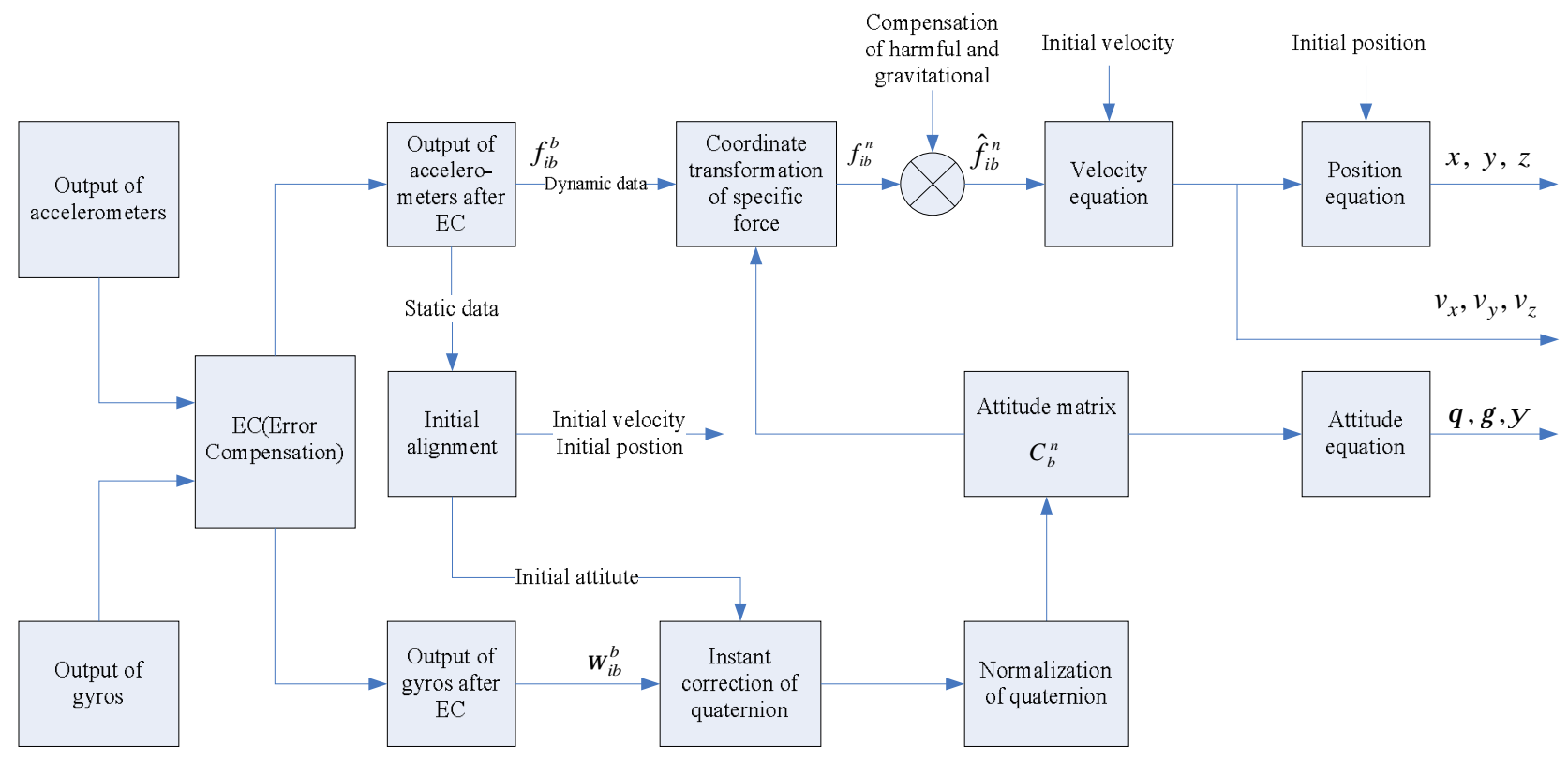

Fig.1: The block diagram of the algorithm of SINS. 


\section{Algorithm model}

\section{Attitude matrix}

The coordinate transformation is the basis of SINS, and the solution of the attitude matrix is the core of the strapdown inertial navigation algorithm. The definition of coordinate frame, attitude angles and attitude matrix are based on literature [4]. And take the geographic coordinate frame as the navigation coordinate frame. The attitude angles equations are derived trough the establishment of the direction cosine matrix from the navigation coordinate frame to the missile-body coordinate frame as shown in Fig.2.

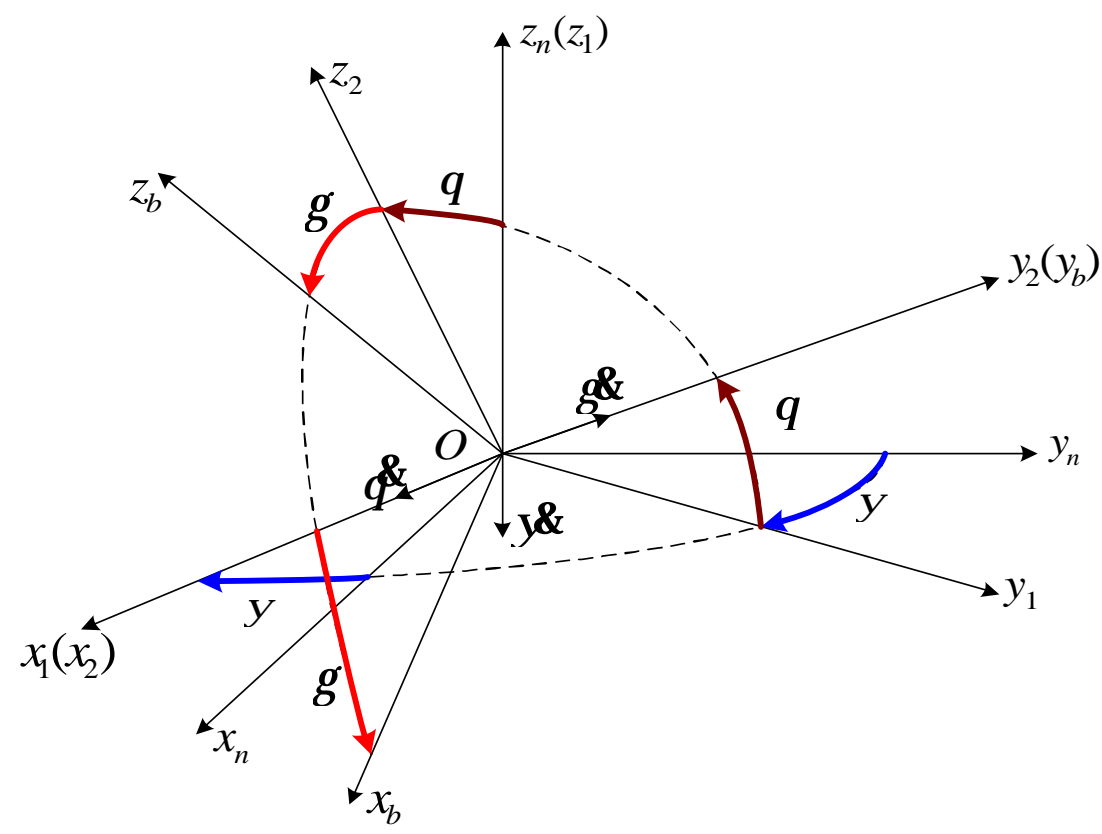

Fig.2: The missile-body coordinate frame is achieved by rotation, from the navigation coordinate frame, in turn of angle $-\psi$ around axis $z_{n}$, angle $\theta$ around axis $x_{1}$ and angle $\gamma$ around axis $y_{2}$.

The direction cosine matrix from the navigation coordinate frame to the missile-body coordinate frame can be represented by equation (1).

$$
\boldsymbol{C}_{n}^{b}=\left[\begin{array}{ccc}
\cos \gamma \cos \psi+\sin \gamma \sin \psi \sin \theta & -\cos \gamma \sin \psi+\sin \gamma \cos \psi \sin \theta & -\sin \gamma \cos \theta \\
\sin \psi \cos \theta & \cos \psi \cos \theta & \sin \theta \\
\sin \gamma \cos \psi-\cos \gamma \sin \psi \sin \theta & -\sin \gamma \sin \psi-\cos \gamma \cos \psi \sin \theta & \cos \gamma \cos \theta
\end{array}\right]
$$

The attitude matrix can be defined as $\boldsymbol{T}$ by equation (2).

$$
\mathbf{T}=\boldsymbol{C}_{b}^{n}=\left(\boldsymbol{C}_{n}^{b}\right)^{\mathrm{T}}=\left[\begin{array}{lll}
T_{11} & T_{12} & T_{13} \\
T_{21} & T_{22} & T_{23} \\
T_{31} & T_{32} & T_{33}
\end{array}\right]
$$

Then, attitude angles are defined by equation (3).

$$
\left\{\begin{array}{c}
\theta=\arcsin \left(T_{32}\right) \\
\gamma=\arctan \left(-T_{31} / T_{33}\right) \\
\psi=\arctan \left(T_{12} / T_{22}\right)
\end{array}\right.
$$

\section{Attitude update model}

Considering the measurement of the angular velocity of the missile is within the missile-body coordinate frame, take quaternion to represent the orientation of the navigation coordinate frame to the missile-body coordinate frame. The quaternion can be represented as equation (4).

$$
\frac{\mathrm{d} \boldsymbol{q}}{\mathrm{d} t}=\frac{1}{2} \boldsymbol{q} \otimes \boldsymbol{\omega}_{n b}^{b}
$$

Where: $\boldsymbol{q}=q_{0}+q_{1} \boldsymbol{i}+q_{2} \boldsymbol{j}+q_{3} \boldsymbol{k}$. 
The attitude matrix can be represented as equation (5).

$$
\mathbf{T}=\left[\begin{array}{ccc}
q_{0}^{2}+q_{1}^{2}-q_{2}^{2}-q_{3}^{2} & 2\left(q_{1} q_{2}-q_{0} q_{3}\right) & 2\left(q_{1} q_{3}+q_{0} q_{2}\right) \\
2\left(q_{1} q_{2}+q_{0} q_{3}\right) & q_{0}^{2}-q_{1}^{2}+q_{2}^{2}-q_{3}^{2} & 2\left(q_{2} q_{3}-q_{0} q_{1}\right) \\
2\left(q_{1} q_{3}-q_{0} q_{2}\right) & 2\left(q_{2} q_{3}+q_{0} q_{1}\right) & q_{0}^{2}-q_{1}^{2}-q_{2}^{2}+q_{3}^{2}
\end{array}\right]
$$

The matrix form of equation (4) is equation (6).

$$
\left[\begin{array}{l}
\phi \\
\phi_{1} \\
\phi_{2} \\
\phi_{3}
\end{array}\right]=\frac{1}{2}\left[\begin{array}{cccc}
0 & -\omega_{n b x}^{b} & -\omega_{n b y}^{b} & -\omega_{n b z}^{b} \\
\omega_{n b x}^{b} & 0 & \omega_{n b z}^{b} & -\omega_{n b y}^{b} \\
\omega_{n b y}^{b} & -\omega_{n b z}^{b} & 0 & \omega_{n b x}^{b} \\
\omega_{n b z}^{b} & \omega_{n b y}^{b} & -\omega_{n b x}^{b} & 0
\end{array}\right]\left[\begin{array}{l}
q_{0} \\
q_{1} \\
q_{2} \\
q_{3}
\end{array}\right]
$$

Where: $\boldsymbol{\omega}_{n b}^{b}$ is the angular velocity of the missile-body coordinate frame around the navigation coordinate frame neglecting the influence the ground velocity and the rotation of the earth.

In order to take fourth order Runge-Kutta method to solve the quaternion, equation (6) should be represented as equation (7).

$$
\phi(t)=\frac{1}{2}\left[\boldsymbol{\omega}_{n b}^{b}(t)\right] \boldsymbol{q}(t)
$$

Take fourth order Runge-Kutta method to solve the differential equation (7), and the initial value of quaternion can be obtained after initial alignment. The quaternion needs to be normalized adopting equation (8).

$$
\boldsymbol{q}=\frac{\tilde{\boldsymbol{q}}}{\sqrt{q_{0}^{2}+q_{1}^{2}+q_{2}^{2}+q_{3}^{2}}}
$$

And after quaternion calculated, angle $\theta$, angle $\gamma$ and angle $\psi$ can be updated by equation (3).

\section{Velocity update model}

In the missile ejection test, the MIMU measurement time is short, and the absolute velocity and position change not obviously, so the update equation of velocity can be represented as equation (9). Generally taking second order Runge-Kutta method to solve equation (9) can meet the precision requirement of velocity.

$$
\mathbf{v}_{n}^{\mathbf{\alpha}}=\boldsymbol{C}_{b}^{n} \boldsymbol{f}^{b}+\mathbf{g}
$$

Where: $v_{n}=\left[\begin{array}{lll}v_{n x} & v_{n y} & v_{n z}\end{array}\right]^{\mathrm{T}}$ is the velocity of missile in the navigation coordinate frame. $f^{b}=\left[\begin{array}{lll}f_{x}^{b} & f_{y}^{b} & f_{z}^{b}\end{array}\right]^{\mathrm{T}}$ is the acceleration of missile in the missile-body coordinate frame. $\boldsymbol{g}=\left[\begin{array}{lll}0 & 0 & g\end{array}\right]^{\mathrm{T}}$ is the acceleration of gravity.

\section{Position update model}

The position can be updated by the integral calculation of equation (10).

$$
\left\{\begin{array}{l}
\&=v_{x}=v_{n x} \\
\&=v_{y}=v_{n y} \\
\&=v_{z}=v_{n z}
\end{array}\right.
$$

\section{Experimental analysis}

In missile ejection test, the measuring device containing MIMU is installed in the missile, and the three axes are corresponding to the three axis of the missile body. After the ejection test, the error compensation of the measure data is compensated, and the static data is used for the initial alignment, and the dynamic data is used to calculate the velocity, position and attitude information of the missile. The missile motion parameters before and after EC (error compensation) are shown in Table 1.

The missile ejection test is carried out under normal temperature environment by adopting cold launching method through a gas generator. The continuous motion of the missile body is about 0.750 
seconds in the catapult, and the missile body will also be subjected to the gas flow for about 0.016 seconds. Based on the data in Table 1, the motion parameters of the missile after EC are closer to the actual measure data than before EC. It shows that the error compensation is effective to the strapdown inertial navigation algorithm.

Table 1: The results of the strapdown inertial navigation algorithm before and after EC.

\begin{tabular}{ccccccc}
\hline & \multicolumn{3}{c}{ The highest point: $t=0.766(\mathrm{~s})$} & \multicolumn{3}{c}{ The maximum position point } \\
\cline { 2 - 6 } & $v_{x \max }(\mathrm{m} / \mathrm{s})$ & $v_{y \max }(\mathrm{m} / \mathrm{s})$ & $v_{z \max }(\mathrm{m} / \mathrm{s})$ & $z_{\max }(\mathrm{m})$ & $y_{\max }(\mathrm{m})$ & $x_{\max }(\mathrm{m})$ \\
\hline Before EC & 0.022 & 5.361 & 40.180 & 95.060 & 47.740 & -0.799 \\
After EC & 0.031 & 5.528 & 39.090 & 88.910 & 45.290 & -0.338 \\
Measured value & 0.029 & 5.525 & 39.100 & 90.030 & 45.814 & -0.501 \\
\hline
\end{tabular}

Fig. 3 and Fig. 4 show the variation curves of the attitude angles before and after the EC respectively. It is clear that the angle $\psi$ and angle $\theta$ are almost the same before and after the EC. But the angle $\gamma$ before EC changes more intense than after EC. On the one hand, the difference is caused by the non compensation; on the other hand, the definition of the attitude angles is also related.

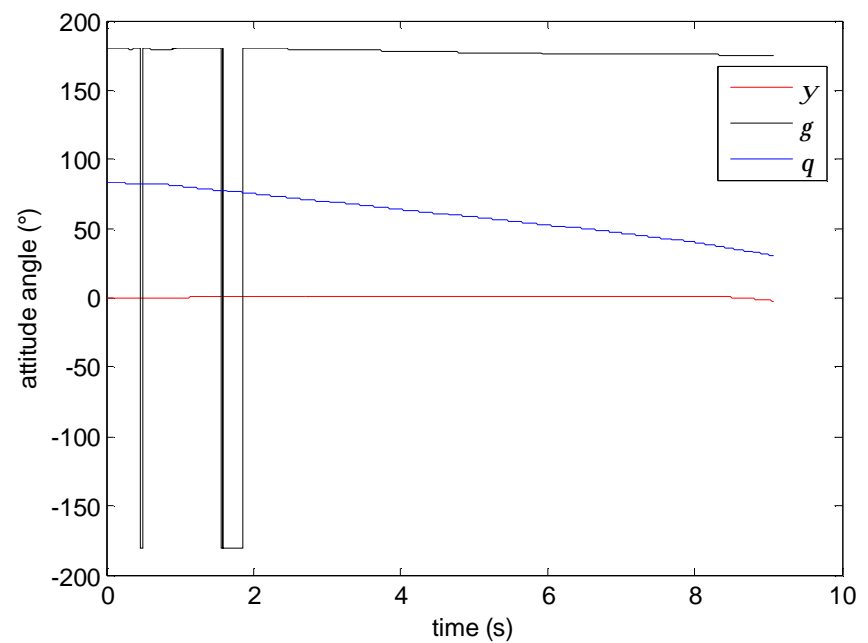

Fig.3: The attitude angles before EC.

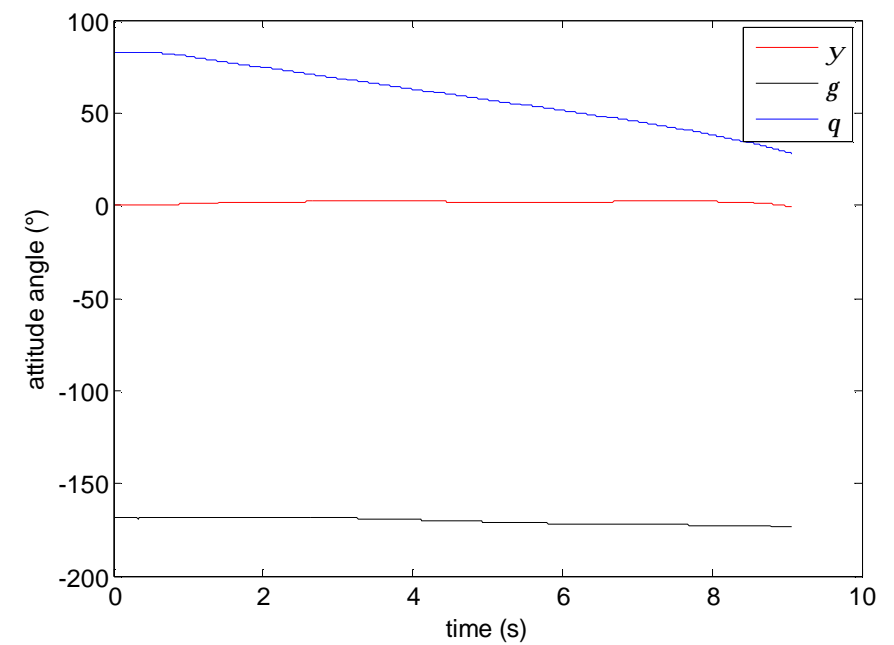

Fig.4: The attitude angles after EC.

\section{Conclusions}

The algorithm discussed is proposed according to the characteristics of missile injection test with short movement time, small changes of velocity and position, and it is based on the principle of SINS and the measurement characteristics of MIMU. The result shows that the algorithm can accurately calculate the position, velocity and attitude angles of the missile. It can meet the measurement requirements in the missile ejection test.

\section{References}

[1] Barbour, N , Hopkins, R , Kourepenis, A , Ward, P, Inertial MEMS System Applications, ADA581018, 2010.

[2] Noureldin, Aboelmagd, Tashfeen B Karamat, and Jacques Georgy, Fundamentals of inertial navigation, satellite-based positioning and their integration, Springer Science \& Business Media, 2012.

[3] Titterton, David and John L Weston, Strapdown inertial navigation technology, volume 17. IET, 2004.

[4] Yongyuan QIN, Inertial Navigation, Science Press, Beijing, 2006. (in Chinese) 